\title{
Acquired Pure Red Cell Aplasia Associated with Chronic Myelomonocytic Leukemia: Too Many of One, Not Enough of the Other
}

\author{
Jose Filipe Gonsalves Ali Bazargan Matthew Ku \\ Department of Clinical Hematology, St Vincent's Hospital, Melbourne, VIC, Australia
}

\section{Keywords}

Anemia - Autoimmunity · Chronic myelomonocytic leukemia - Immunology · Pure red cell aplasia

\begin{abstract}
There is a growing body of literature outlining the association between certain hematological malignancies, such as chronic myelomonocytic leukemia (CMML), and systemic autoimmune diseases. Diagnosis and management can be difficult, particularly when autoimmune phenomena overlap with features of the underlying illness. This is especially the case in patients who develop immune-mediated cytopenias in the context of underlying bone marrow disease. CMML associated with immune thrombocytopenia and hemolytic anemia has been reported a number of times in the literature; however, there are only scattered case reports describing CMML associated with acquired pure red cell aplasia. Here, we describe the diagnostic and management approach to a patient who developed both diseases.
\end{abstract}

(c) 2020 The Author(s).

Published by S. Karger AG, Basel

\section{Introduction}

Chronic myelomonocytic leukemia (CMML) is a hematological malignancy characterized by the proliferation of bone marrow leukocytic precursors resulting in peripheral monocytosis, as well as underlying dysplasia with a tendency to transform to acute myeloid leukemia. It is the most common subtype of the myelodysplastic (MDS) and myeloproliferative neoplasm (MPN) overlap syndromes and has markedly a heterogenous clinical phenotype [1]. There is increasing recognition of the impact that CMML has on the patient's immune milieu [2-4]. 
A number of systemic autoimmune and inflammatory diseases may arise in the context of a deranged immunological environment. One such entity is idiopathic acquired pure red cell aplasia (aPRCA). This rare condition is thought to be mediated by selective targeting of erythroid precursors by autoreactive immune effector cells [5]. Patients typically present with severe anemia while bone marrow examination is often characterized by a complete absence of erythropoiesis.

There have only been isolated case reports of aPRCA associated with CMML. While several distinct forms of aPRCA are recognized, secondary aPRCA in particular has a strong association with hematological malignancies [5]. Most published reports of CMML-associated aPRCA have demonstrated at least a partial response to immunosuppressive medications [6, 7]. Therefore, it is likely that, in a significant proportion of these patients, aPRCA occurs in the context of profound immune dysregulation.

The optimal treatment strategy for these rare patients is difficult to define. For example, it remains uncertain as to whether immunosuppression to control immune-driven PRCA might accelerate transformation of CMML to acute leukemia. At least one case report suggested a potential causative role for these medications in transformed disease [6]. It is also unclear whether disease modifying therapies used to treat CMML, such as azacitidine, might ameliorate bystander immune epiphenomena if indeed they are related. There is some support for this approach in the literature [8]. Here we report on the successful treatment of a patient with CMML, who had associated PRCA on bone marrow aspirate and trephine.

\section{Case Report}

In February 2017, a 76-year-old man presented with a 3-week history of tiredness, malaise and dyspnea on exertion. He was previously fit and active with a past history of skin basal cell and squamous cell carcinomas (SCC) that required surgical resections. He also had a history of hypertension which was treated with perindopril. He was an ex-smoker with limited alcohol intake. The reported symptoms followed a previous upper respiratory tract infection; however, they became persistent despite resolution of the infection. There were no B-symptoms or other associated features. Examination was unremarkable with no evidence of lymphadenopathy or hepatosplenomegaly.

The blood tests conducted by the family physician showed evidence of pancytopenia with a concurrent monocytosis, which represented a significant change from the previous normal results. The hemoglobin was $9.7 \mathrm{~g} / \mathrm{dL}, \mathrm{MCV} 115 \mathrm{fL}$, white blood cell count $3.4 \times 10^{9} / \mathrm{L}$, neutrophils $0.6 \times 10^{9} / \mathrm{L}$, monocytes $1.6 \times 10^{9} / \mathrm{L}$ and platelet count $103 \times 10^{9} / \mathrm{L}$. The reticulocyte level was $8 \times 10^{9} / \mathrm{L}$, consistent with a poor marrow response to the anemia. The blood film showed rare dysplastic neutrophils, but no blasts were seen. Viral serologies such as HIV, hepatitis B and $\mathrm{C}$ were negative. Parvovirus B19 was also negative. Autoimmune screen, including antinuclear antibodies, and hemolytic screen were unremarkable. Vitamin $\mathrm{B}_{12}$, folate, iron studies and thyroid function tests were also within the normal range. The erythropoietin level was significantly elevated at 1,107.8 U/L. Chest imaging did not reveal a thymoma. After a fortnight, the repeat blood tests showed worsening cytopenias with the hemoglobin level deteriorating to $7 \mathrm{~g} / \mathrm{dL}$, necessitating red blood cell transfusions due to symptom exacerbation. The repeat blood film showed a leukoerythroblastic picture. Bone marrow aspirate and trephine showed multilineage dysplastic features consistent with CMML, but also identified an absence of erythroid precursors indicating PRCA. The cytogenetics showed a loss of the $Y$ chromosome, a common finding in older males in the absence of a hematological malignancy.

The patient was initially treated with high-dose prednisolone and cyclosporine. As the dose of cyclosporine was titrated up, the prednisolone dose was able to be weaned down. In

\section{Karger'}


the first 10 months, the patient maintained a hemoglobin of around $10 \mathrm{~g} / \mathrm{dL}$, albeit with occasional transfusions. He maintained good energy levels and carried out his usual daily activities independently. Initially, he did not develop any significant toxicities from the immunosuppression such as renal impairment or infections. At this stage, the patient did not qualify for azacitidine treatment under our Pharmaceutical Benefits Scheme. Ten months later, treatment was complicated by a new localized right temporal SCC. This was surgically resected followed by adjuvant radiotherapy. Cyclosporine was ceased due to its potentiating effect on skin cancers. Repeat bone marrow aspirate and trephine again showed marked hypercellularity with near-complete absence of erythropoiesis. However, this time, multilineage dysplasia was accompanied by an excess blast count, consistent with CMML-2. Due to progression of disease, the patient became eligible for azacitidine and this was commenced with the hope that treatment of CMML would improve the associated PRCA. There were no new cytogenetic findings. Iron chelation was also administered due to evidence of transfusion-related iron overload.

Despite 6 months of treatment with azacitidine, there was worsening anemia with escalating transfusion requirements. A further repeat bone marrow aspirate and trephine demonstrated ongoing multilineage dysplastic changes in the granulocytic and megakaryocytic series. No excess of blasts was seen, ostensibly due to the treatment effect of azacitidine on the CMML. This was reflected by the improvement of neutropenia and thrombocytopenia. However, even with improvement in CMML, there continued to be an absence of erythropoiesis. After much discussion, the decision was made to rechallenge the patient with cyclosporine given its previous efficacy. The patient was thoroughly checked for any suspicious skin lesions. He was also commenced on acitretin $10 \mathrm{mg}$ daily as chemoprophylaxis for SCC. The combination of azacitidine and cyclosporine proved efficacious in reducing the patient's transfusion requirement and, after 12 months of acitretin treatment, there has been no emergence of new skin cancers. Three years from diagnosis, the patient is well and receiving ongoing reviews.

\section{Discussion}

With an incidence of between 10 and 20\%, the immunological sequelae of CMML and MDS are now appreciated to be a significant source of morbidity in this population [4] A recent French retrospective study demonstrated significant heterogeneity in the nature of systemic inflammatory diseases associated with CMML/MDS [3]. Notably the authors identified that CMML/MDS patients with systemic inflammatory and autoimmune diseases were more likely to have adverse risk prognostic features than controls. A subsequent study focusing on CMML patients again found that those patients with immune epiphenomena were more likely to have unfavorable disease-related features [2].

Franzini et al. [9] demonstrated that the transcriptional signature of monocytes derived from CMML patients is particularly pro-inflammatory, with increases in tumor-necrosis factor and interleukin- 6 signaling amongst others. They also raise the interesting prospect of whether anti-inflammatory therapies may play a role in the management of CMML patients.

In our patient, there was a rapid improvement in reticulocytopenia following the introduction of high dose immunosuppression. Unfortunately, the development of non-melanoma skin cancers is a well-recognized side effect of agents such as cyclosporine, and in this case necessitated treatment interruption.

After demonstrating evolution of the underlying disease process, we had hoped that instituting CMML-directed therapy would also afford a degree of control over the patient's aPRCA. The efficacy of this approach was demonstrated by Fraison et al. [8] in 2015. They

\section{Karger ${ }^{\prime \prime}=$}


found that azacitidine was effective in controlling secondary autoimmune disease in patients with MDS/CMML [8]. Unfortunately, as the patient did not respond, we had to revisit the requirement for immunosuppression.

The decision to reintroduce immunosuppression was taken only after careful consideration of the potential hazards. For example, studies have demonstrated a link between immunosuppression for solid organ transplantation and the development of myeloid malignancies such as MDS and acute myeloid leukemia [10]. Given the tendency for CMML to transform to acute leukemia, we were mindful of the theoretical potential for immunosuppression to provoke further evolution of the patient's CMML.

While cyclosporine has been implicated as a potential driver for acute leukemia transformation, a 2017 retrospective case-control study of over 40,000 patients with autoimmune disease was not able to implicate it as a risk factor for myeloid malignancies [11]. Furthermore cyclosporine, in conjunction with antithymocyte globulin, has been investigated as a potential therapy for myelodysplastic syndromes, with evidence of efficacy in some subsets of the disease [12]. As our patient had clearly derived benefit from cyclosporine previously, it was decided that a rechallenge with the same agent was appropriate.

Given the patient had demonstrated a propensity toward developing SCC whilst on immunosuppression, chemoprophylaxis with acitretin was felt to be prudent, especially given encouraging results with this agent in solid organ transplant recipients [13].

Our case highlights the difficulties in managing patients with CMML who develop a secondary auto-immune disease such as PRCA. As this is a complex condition with a number of immunological factors, further research is necessary to identify optimal treatment approaches going forward.

\section{Statement of Ethics}

The patient described in the manuscript has given their informed consent regarding the publication of this case.

\section{Conflict of Interest Statement}

The authors have no conflicts of interest to declare.

\section{Funding Sources}

There are no funding sources to declare.

\section{Author Contributions}

M.K. and A.B. provided the case and were involved in managing the patient. M.K. wrote the case report and serves as the corresponding author. J.F.G. wrote the introduction and discussion.

\section{Karger' ${ }^{\prime}=$}




\section{References}

1 Solary E, Itzykson R. How I treat chronic myelomonocytic leukemia. Blood. 2017;130(2):126-36.

2 Grignano E, Mekinian A, Braun T, Liozon E, Hamidou M, Decaux 0, et al. Autoimmune and inflammatory diseases associated with chronic myelomonocytic leukemia: A series of 26 cases and literature review. Leuk Res. 2016;47:136-41.

3 Mekinian A, Grignano E, Braun T, Decaux O, Liozon E, Costedoat-Chalumeau N, et al. Systemic inflammatory and autoimmune manifestations associated with myelodysplastic syndromes and chronic myelomonocytic leukaemia: A French multicentre retrospective study. Rheumatology (Oxford). 2015;55(2):291-300.

4 Ambinder AJ, Miller J, DeZern AE. Autoimmune disease in CMML-the chicken or the egg? Best Pract Res Clin Haematol. 2020 Jun;33(2):101136.

5 Balasubramanian SK, Sadaps M, Thota S, Aly M, Przychodzen BP, Hirsch CM, et al. Rational management approach to pure red cell aplasia. Haematologica. 2018 Feb;103(2):221-230.

6 Tanna S, Ustun C. Immunosuppressive treatment in patient with pure red cell aplasia associated with chronic myelomonocytic leukemia: harm or benefit? Int J Hematol. 2009 Dec;90(5):597-600.

7 Williamson PJ, Oscier DG, Bell AJ, Hamblin TJ. Red cell aplasia in myelodysplastic syndrome. J Clin Pathol. 1991. 1991;44(5):431-2.

8 Fraison JB, Mekinian A, Grignano E, Kahn JE, Arlet JB, Decaux O, et al. Efficacy of Azacitidine in autoimmune and inflammatory disorders associated with myelodysplastic syndromes and chronic myelomonocytic leukemia. Leuk Res. 2016;43:13-7.

9 Franzini A, Pomicter AD, Yan D, Khorashad JS, Tantravahi SK, Than H, et al. The transcriptome of CMML monocytes is highly inflammatory and reflects leukemia-specific and age-related alterations. Blood Adv. 2019 Oct 22;3(20):2949-61.

10 Morton LM, Gibson TM, Clarke CA, Lynch CF, Anderson LA, Pfeiffer R, et al. Risk of myeloid neoplasms after solid organ transplantation. Leukemia. 2014;28(12):2317-23.

11 Ertz-Archambault N, Kosiorek H, Taylor GE, Kelemen K, Dueck A, Castro J, et al. Association of therapy for autoimmune disease with myelodysplastic syndromes and acute myeloid leukemia. JAMA Oncol. 2017;3(7): 936-43.

12 Götze K, Platzbecker U. Old dogs, new tricks: revisiting immune modulatory approaches for myelodysplastic syndromes. HemaSphere. 2018 Nov 29;2(6):e162.

13 Otley CC, Stasko T, Tope WD, Lebwohl M. Chemoprevention of nonmelanoma skin cancer with systemic retinoids: Practical dosing and management of adverse effects. Dermatol Surg. 2006;32(4):562-8. 